Check for updates

Cite this: RSC Adv., 2018, 8, 16278

\title{
Interspecies comparison in the COMT-mediated methylation of 3-BTD†
}

\begin{abstract}
Yangliu Xia, (D) ${ }^{a}$ Huilin Pang, ${ }^{a}$ Tongyi Dou, ${ }^{\star a}$ Ping Wang ${ }^{\star b}$ and Guangbo Ge*b
Catechol-O-methyltransferase (COMT) is a druggable biological target and COMT modulators have been widely applied in the treatment of various central and peripheral nervous system disorders. The interspecies differences of COMT were carefully investigated using 3-BTD (a newly developed fluorescent probe of COMT) methylation as the probe reaction, and liver S9 from humans and seven experimental animals including monkeys, dogs, mice, rats, minipigs, guinea pigs and New Zealand rabbits as the enzyme source. Metabolite profiling demonstrated that all the tested liver 59 samples from the different animals could catalyse 3-BTD methylation but displayed significant differences in reaction rate. Also, the differential effects of tolcapone (a potent inhibitor against COMT) on 3-BTD methylation among various species were observed. The apparent kinetic parameters and the maximum intrinsic clearances $\left(\mathrm{Cl}_{\text {int }}\right)$ for 3-BTD methylation in liver S9 from the different animals were determined, and the order of the $\mathrm{Cl}_{\text {int }}$ values for the formation of 3-BTD was RLS9 > DLS9 $\approx$ PLS9 > MLS9 > CyLS9 > RaLS9 > GpLS9 > HLS9. These findings are helpful for further exploring COMT-associated biological processes in animal models, as well as for developing therapeutic molecules that target COMT.
\end{abstract}

Received 5th March 2018

Accepted 18th April 2018

DOI: $10.1039 / \mathrm{c} 8 \mathrm{ra01938j}$

rsc.li/rsc-advances catecholic drugs, dietary catechols and catechol estrogens. ${ }^{\mathbf{8}, 9}$ Notably, both the expression and function of COMT can be affected by genetic polymorphisms and a variety of phytochemicals and therapeutic drugs, as well as environmental and pathological factors. ${ }^{10,11}$ However, limited by the lack of an available COMT probe with high sensitivity and good reactivity, it is still difficult to identify and characterize COMT modulators, as well as to investigate the functional changes in physiological and pathological states.

3-BTD (3-(benzo[ $d]$ thiazol-2-yl)-7,8-dihydroxy-2H-chromen-2one) is a newly developed two-photon fluorescent probe of COMT that has been successfully used for the rapid, selective and sensitive detection of COMT in complex biological samples (Fig. 1). ${ }^{12}$ With good cell permeability, low cytotoxicity, and high imaging resolution, 3-BTD could also be used in the bioimaging of endogenous COMT in living cells and tissue slices. ${ }^{12}$ Overall, 3-BTD displays high efficiency for measuring the real activities of COMT, and offers a possibility for exploring related diseases. ${ }^{6,7}$ Furthermore, COMT is also responsible for the metabolic elimination of xenobiotic substances and hormones incorporating catecholic structures, including

${ }^{a}$ Laboratory of Pharmacology \& Toxicology, School of Life Science and Medicine, Dalian University of Technology, Panjin 124221, China. E-mail: douty@dlut.edu.cn ${ }^{b}$ Shanghai University of Traditional Chinese Medicines, Shanghai, 201203, China. E-mail:s200541025@126.com; gegbdicp@sohu.com

$\dagger$ Electronic supplementary information (ESI) available: ${ }^{1} \mathrm{H}$ and ${ }^{13} \mathrm{C}$ NMR, HSQC, HMBC and MS spectra of 3-BTMD, and MS spectrum of 3-BTD; percent identity matrix of similarity of amino acid sequences between COMT from different species; phylogenetic tree of catechol-O-methyl transferases (COMT) from different species. See DOI: 10.1039/c8ra01938j 
COMT-associated biological processes and functions in living systems, as well as for developing therapeutic molecules that target COMT.

Animal models are indispensable tools for use in further studies of the pharmacological and physiological roles of COMT, and must be used in the pharmacological and toxicological testing of a drug candidate targeting COMT. ${ }^{\mathbf{1 3 - 1 5}}$ The interspecies similarities and differences of COMT, in terms of the kinetic behaviours and the response towards a modulator(s), have not been systematically investigated, limiting the understanding of the potential role of COMT in the origin and progression of certain diseases, and the development of COMT modulators as therapeutic agents. In order to explore the interspecies differences of COMT, 3-BTD methylation was used as the probe reaction, and liver S9 from humans and commonly used animal species including cynomolgus monkeys, dogs, mice, rats, minipigs, guinea pigs and New Zealand rabbits was used as the enzyme source. The methylation behaviour of 3-BTD in liver S9 from these different species was characterized with respect to the similarities and differences of their metabolic profiles, catalytic efficacy and inhibitory potency by a known chemical inhibitor.

\section{Results}

\section{3-BTD methylation in liver S9 from the different animal species}

The metabolic profiles of 3-BTD methylation in liver S9 from mice, rats, dogs, minipigs, monkeys, New Zealand rabbits, guinea pigs and humans $(0.5 \mathrm{mg}$ protein per $\mathrm{ml})$ were plotted, following $30 \mathrm{~min}$ incubation under physiological conditions, and it was evident that 3-BTD could be methylated in all the tested liver S9 samples (Fig. 2). The methylated metabolite was biosynthesized using mixed liver S9 samples from the different species as the enzyme source, and the 8-O-methylated product of 3-BTD was also chemically synthesized and fully characterized as 3-BTMD using ${ }^{1} \mathrm{H}$ NMR, ${ }^{13} \mathrm{C}$ NMR, HSQC, HMBC and MS spectra. The characteristic proton signal of a C-7 hydroxyl was

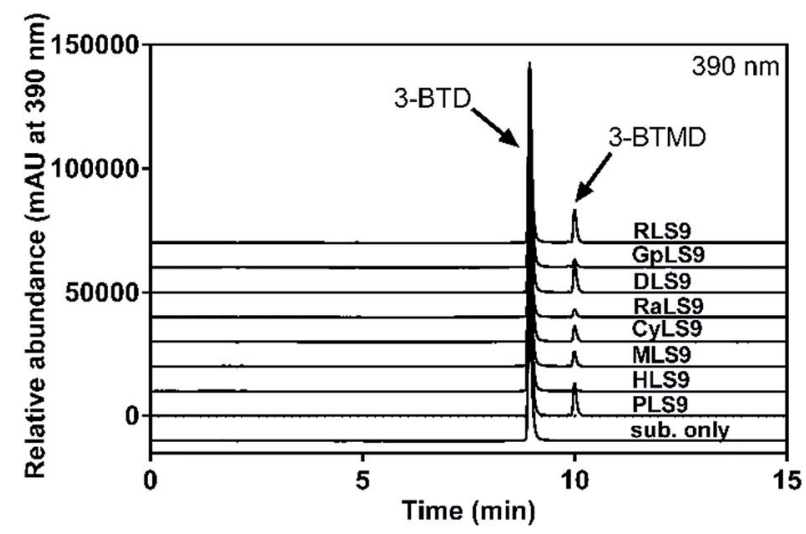

Fig. 2 Representative LC-UV profiles of 3-BTD and its methylated metabolite in liver $\mathbf{S 9}$ from humans, monkeys, dogs, mice, rats, minipigs, guinea pigs and New Zealand rabbits. 3-BTD $(10 \mu \mathrm{M})$ was incubated with liver $\$ 9\left(0.5 \mathrm{mg} \mathrm{ml}^{-1}\right)$ from each species at $37^{\circ} \mathrm{C}$ for $30 \mathrm{~min}$. observed at $10.9 \mathrm{ppm}$ in the ${ }^{1} \mathrm{H}$ NMR spectra of both the chemand bio-synthesized products, and the lack of any signal ( $\sim 9.5$ ppm) that may be assigned to a C-8 proton provided additional evidence against C-7 methylation. With the help of an 8-Omethylated standard, the methylated metabolite formed in the methylation systems was confirmed to be the 8-O-methylated product, 3-BTMD. The ${ }^{1} \mathrm{H}$ and ${ }^{13} \mathrm{C}$ NMR, HSQC and HMBC spectra of the chem-synthesized product are displayed in the ESI (Fig. S1-S5 $\dagger$ ), and the ${ }^{1} \mathrm{H}-\mathrm{NMR}$ of the bio-synthesized product is also provided (Fig. S6 $\dagger$ ). The formation of the metabolite in all the tested liver S9 samples was time- and S9dependent. The formation of 3-BTMD in liver S9 from the different animal species was faster than that in HLS9, while PLS9 displayed the fastest methylated rate of 3-BTD among all the tested liver S9 samples (Fig. 2).

\section{Kinetic analyses of 3-BTD methylation in liver S9 from the various species}

Since substrate affinity and catalytic efficiency are essential for evaluating the species differences of a given biotransformation, the kinetic parameters for 3-BTD methylation in liver S9 from various species were determined. As shown in Fig. 3, the kinetic plots of 3-BTD methylation in liver S9 from all the tested animal
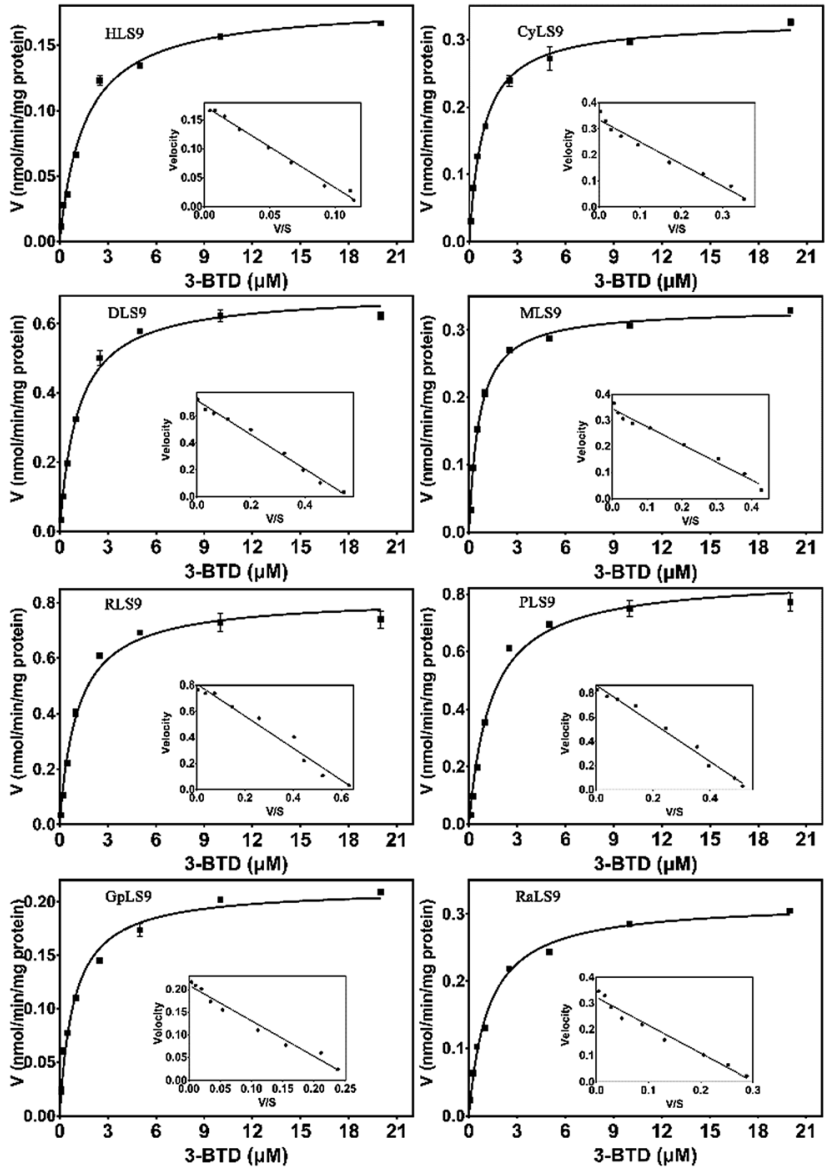

Fig. 3 Enzymatic kinetic plots of 3-BTD methylation in liver S9 from the different species. 3-BTD $(0.1-20 \mu \mathrm{M})$ was incubated with HLS9, CyLS9, DLS9, MLS9, RLS9, PLS9, GpLS9 or RaLS9 at $37^{\circ} \mathrm{C}$. 
Table 1 Kinetic parameters for the formation of 3-BTMD in HLS9, CyS9, DLS9, MS9, RS9, PLS9, GpLS9 and RaLS9. $K_{m}$ and $V_{\max }$ values are expressed as best fit values \pm S.E.

\begin{tabular}{lcll}
\hline $\begin{array}{l}\text { Enzyme } \\
\text { source }\end{array}$ & $K_{\mathrm{m}}(\mu \mathrm{M})$ & $\begin{array}{l}V_{\max } \\
\left(\mathrm{nmol} \mathrm{min} \mathrm{mg}^{-1}\right)\end{array}$ & $\begin{array}{l}\mathrm{Cl}_{\text {int }} \\
\left(\mu 1 \mathrm{~min}^{-1} \mathrm{mg}^{-1}\right)\end{array}$ \\
\hline HLS9 & $1.6 \pm 0.31$ & $0.18 \pm 0.0057$ & 112 \\
CyLS9 & $0.89 \pm 0.059$ & $0.33 \pm 0.0055$ & 370 \\
DLS9 & $1.1 \pm 0.085$ & $0.69 \pm 0.013$ & 627 \\
MLS9 & $0.63 \pm 0.030$ & $0.33 \pm 0.0037$ & 523 \\
RLS9 & $1.1 \pm 0.15$ & $0.84 \pm 0.029$ & 763 \\
PLS9 & $1.4 \pm 0.13$ & $0.86 \pm 0.022$ & 614 \\
GpLS9 & $1.2 \pm 0.18$ & $0.23 \pm 0.0092$ & 191 \\
RaLS9 & $1.4 \pm 0.13$ & $0.33 \pm 0.0083$ & 235 \\
& & &
\end{tabular}

species are well-fitted with the Michaelis-Menten equation. The apparent kinetic parameters including $K_{\mathrm{m}}$, and the $V_{\max }$ of 3-BTD methylation were determined (Table 1). Notably, the $K_{\mathrm{m}}$ values for the formation of 3-BTMD in liver S9 from the seven animal species were close to the $K_{\mathrm{m}}$ value in HLS9, implying that 3-BTD displayed similar substrate affinity towards the mammalian COMT from the different species. However, the reactivity $\left(V_{\max }\right)$ towards 3-BTD methylation in liver S9 from the various species ranged from 0.18 to 0.86 nmol per min per mg protein. Consequently, the $\mathrm{Cl}_{\text {int }}$ values for the formation of 3-BTMD varied greatly among the eight species, with a range of $112-763 \mu \mathrm{min}^{-1} \mathrm{mg}^{-1}$. The order of the $\mathrm{Cl}_{\text {int }}$ values for the formation of 3-BTD was RLS9 $>$ DLS9 $\approx$ PLS9 > MLS9 > CyLS9 > RaLS9 > GpLS9 > HLS9, which was consistent with the trend of the methylated profiles of 3-BTD in these enzyme sources.
Inhibitory effects of tolcapone on 3-BTD methylation in liver S9 from the various species

Next, tolcapone (a potent inhibitor of COMT) was used to further characterize the similarity of 3-BTD methylation in liver S9 from humans and seven animal species. The dose-dependent inhibition curves and determined $\mathrm{IC}_{50}$ values of tolcapone on 3BTD methylation in the liver S9 are depicted in Fig. 4 and Table 2. Among all the tested liver S9, tolcapone showed the most potent inhibition towards 3-BTD methylation in HLS9, with an $\mathrm{IC}_{50}$ value of $17.0 \mathrm{nM}$. A comparison of the $\mathrm{IC}_{50}$ values indicated that the inhibitory potency of tolcapone on 3-BTD methylation in liver S9 from the animals versus HLS9 varied between 1.1-fold (CyLS9) and 6.9-fold (RaLS9). This striking variation in the inhibitory effect of tolcapone demonstrated that the mammalian COMT in liver S9 from the different animals was less sensitive towards tolcapone in contrast to the sensitivity of the human liver COMT. These results indicate that tolcapone is a more sensitive and selective inhibitor against human COMTmediated 3-BTD methylation, but that this inhibitor is less sensitive towards the COMT of the examined animal species.

\section{Discussion}

As the enzyme responsible for the $O$-methylation of endogenous neurotransmitters involved in many biological functions, the modulation of the COMT enzyme has become an attractive strategy for manipulating the levels of endogenous neurotransmitters such as dopamine, epinephrine, and norepinephrine. ${ }^{16-20}$ The promising application of COMT inhibitors in the treatment of various central and peripheral nervous system disorders has attracted increasing attention from researchers in
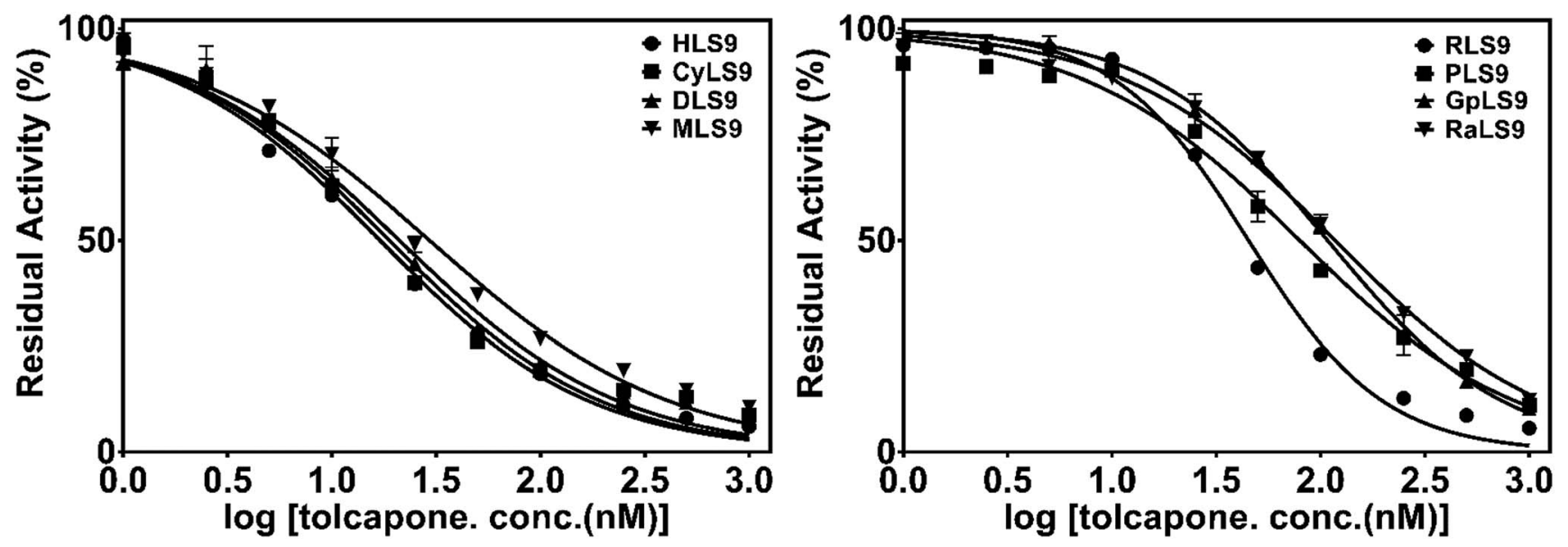

Fig. 4 The dose-dependent inhibition curves of tolcapone on 3-BTD methylation in liver S9 from the different species.

Table 2 IC 50 values of tolcapone towards COMT in HLS9, CyS9, DLS9, MS9, RS9, PLS9, GpLS9 and RaLS9, using 3-BTD-8-O-methylation as the probe reaction. Data are presented as mean \pm S.E. of the computer calculation

\begin{tabular}{|c|c|c|c|c|c|c|c|c|}
\hline $\mathrm{IC}_{50}(\mathrm{nM})$ & $17.0 \pm 4.4$ & $18.7 \pm 3.0$ & $20.8 \pm 2.7$ & $29.0 \pm 3.9$ & $45.2 \pm 5.1$ & $79.6 \pm 11.5$ & $108.3 \pm 7.1$ & $117.3 \pm 10.3$ \\
\hline
\end{tabular}


academia and the pharmaceutical industry. ${ }^{21}$ However, the similarities or differences in the function of COMT among various species and their responses towards a COMT inhibitor(s) have been rarely reported. It is well known that whole body tests in laboratory animals are always needed in preclinical studies for drug discovery and development, and that the animal data are commonly extrapolated to humans. ${ }^{22-25}$ Thus, choosing a suitable laboratory animal(s), in which the tissue distribution, functions and biological responses of COMT are similar to those of COMT in humans, is crucial for in vivo testing of a given compound targeting COMT.

More recently, we have developed a novel two-photon fluorescent probe for COMT that exhibits high specificity and good reactivity, and that is applicable for monitoring the real activities of COMT in vitro. ${ }^{12}$ The good properties and application potentials of 3-BTD in complex biological systems motivated us to use this probe as a novel tool to explore the difference in the function of COMT among the various species and their responses towards a known COMT inhibitor(s). This will be very helpful for choosing the most suitable laboratory animal(s) for investigating the pharmacokinetic behaviors of COMT modulators and COMT-associated biological processes. To this end, the interspecies differences in the metabolic behaviors of 3-BTD methylation among the eight species and their responses towards a COMT inhibitor were carefully investigated in the present study.

The metabolic profiling study revealed that 3-BTD methylation could be catalyzed by liver $\mathrm{S} 9$ from all the examined species including humans, cynomolgus monkeys, dogs, mice, rats, minipigs, guinea pigs and New Zealand rabbits (Fig. 2). Furthermore, kinetic analyses demonstrated that 3-BTD methylation in liver $\mathrm{S} 9$ from all the tested animal species followed similar kinetics (Michaelis-Menten), with similar $K_{\mathrm{m}}$ values ranging from $0.63 \mu \mathrm{M}$ to $1.6 \mu \mathrm{M}$ (Table 1 ). These findings suggested that 3-BTD displayed similar substrate affinity towards the mammalian COMT from the different species. However, the $V_{\text {max }}$ values of 3-BTD methylation in liver S9 from all the tested animals were altered significantly probably due to the different expression levels of COMT in animal livers. These results also agree well with a previous study, in which the COMT in a dog liver was found to be much more active than the COMT in the liver of a rabbit but was roughly equipotent to the COMT obtained from a rat and a monkey. ${ }^{26}$

Tolcapone is a high affinity nitro catechol type inhibitor of catechol-O-methyl transferase (COMT) and is commonly used as an adjunct in the treatment of Parkinson's disease (PD). ${ }^{27}$ It was evident from Fig. 4 that tolcapone at a dose of $1000 \mathrm{nM}$ could strongly inhibit mammalian COMT-mediated 3-BTD methylation in liver S9 from all the test species, but that the $\mathrm{IC}_{50}$ values varied greatly (17.0-117.3 nM). The nanomolar $\mathrm{IC}_{50}$ values are quite consistent with the reported $\mathrm{IC}_{50}$ values of tolcapone when using different substrates including adrenaline, epinephrine or 3,4-dihydroxybenzoic acid. ${ }^{28-30}$ The $\mathrm{IC}_{50}$ value of tolcapone against 3-BTD methylation in CyLS9 was the most similar to the value in HLS9, and the response of 3-BTD methylation towards tolcapone in CyLS9 was more sensitive than the response in liver S9 from the other laboratory animals, including dogs, mice, rats, minipigs, guinea pigs and New Zealand rabbits. Among all the studied animal species, the monkey COMT shared the highest similarity with the human COMT, with an amino acid sequence homology of $91.85 \%$ (ESI Fig. S7 and Table S1 $\dagger$ ). It is worth mentioning that the inhibitory potency $\left(\mathrm{IC}_{50}\right.$ value) of tolcapone in liver $\mathrm{S} 9$ from the various animals agreed well with the COMT amino acid sequence homology between humans and different animals (ESI Fig. S7 and Table S1 $\dagger$ ). In terms of the amino acid sequence homology and the effects of tolcapone on 3-BTD methylation, the COMT from cynomolgus monkeys is the most similar ortholog of the COMT from humans. To further explore the similarities in the function of mammalian COMT, more indepth studies on the effects of other COMT inhibitors against the methylation of 3-BTD and other COMT substrates should be conducted.

It is well known that COMT is a therapeutic target for the treatment of various central and peripheral nervous system disorders, and increasing interest has been seen in the development of COMT inhibitors. ${ }^{20,31}$ At present, the identification and characterization of COMT inhibitors are usually conducted in vitro using recombinant COMT or tissue fractions as enzyme sources. It should be pointed out that the in vivo potency of COMT inhibitors should be assessed in laboratory animals during preclinical studies. However, despite several reports considering the differences in the COMT amino acid sequences or the enzyme activities from different species, ${ }^{26,32-34}$ comprehensive information about the differences in the kinetic behaviours and the responses towards an inhibitor(s) of COMT between humans and other species has rarely been reported, and this may be quite helpful in animal model selection and data interpretation when conducting in vivo evaluation of COMT inhibitors. In the present study, 3-BTD methylation was used as a COMT probe reaction for exploring the potential similarities or differences in the function of COMT from various animals. The interspecies differences of COMT were carefully investigated using 3-BTD methylation as the probe reaction and liver $\mathrm{S} 9$ from various animals as the enzyme source, with respect to the metabolic profiles, the response of a COMT inhibitor, and the enzymatic kinetics. Taken together, our results demonstrate that the responses towards a chemical inhibitor vary greatly among various animals, and the COMT from cynomolgus monkeys displayed the most similar response towards tolcapone compared to the COMT in HLS9.

\section{Conclusion}

In summary, the interspecies differences of COMT were investigated using 3-BTD methylation as the probe reaction and liver S9 from different animal species as the enzyme source, with respect to the similarities in metabolic profiles, kinetic behaviors and the response towards a known COMT inhibitor. Kinetic analyses showed that 3-BTD methylation in liver S9 from all the tested species displayed similar kinetic behaviors (MichaelisMenten) and substrate affinity, but the reactivity and $\mathrm{Cl}_{\text {int }}$ varied among the various species. Furthermore, the inhibitory effects of tolcapone (a potent inhibitor against COMT) on 3-BTD 
methylation was investigated in the liver S9 from the different species, and the responses towards this chemical inhibitor varied greatly among the various laboratory animals. All these findings are very helpful for further exploring COMT-associated biological processes in animal models, as well as for developing therapeutic molecules that target COMT.

\section{Experimental}

\section{Materials}

3-BTD (3-(benzo[d]thiazol-2-yl)-7,8-dihydroxy-2H-chromen-2one) and 3-BTMD (3-(benzo[d]thiazol-2-yl)-7-hydroxy-8methoxy-2H-chromen-2-one) were synthesized by the authors, and the purity was above $98 \%$ as determined using HPLC-UV. Magnesium chloride $\left(\mathrm{MgCl}_{2}\right)$, DL-dithiothreitol (DTT), $S$-(5'adenosyl)-L-methionine- $p$-toluenesulfonate salt (SAM) and tolcapone were purchased from Sigma-Aldrich (St. Louis, MO, USA). The stock solutions of these compounds were freshly prepared in DMSO and stored at $4{ }^{\circ} \mathrm{C}$ until use.

Liver S9 samples of male cynomolgus monkeys (CyLS9, Lot. AVDR), male beagle dogs (DLS9, Lot. LKMH), male ICR/CD-1 mice (MLS9, Lot. NEJZ), male Sprague-Dawley rats (RLS9, Lot. GKXW), male Yucatan minipigs (PLS9, Lot. WZZS), DunkinHartley guinea pigs (GpLS9, Lot. ZUSV-1), New Zealand rabbits (RaLS9, Lot. QRCI) and male Mongolian humans (HLS9, Lot. PBCQ) were purchased from the Research Institute for Liver Diseases (RILD, Shanghai, China). All microsomal samples were stored at $-80{ }^{\circ} \mathrm{C}$ until use.

\section{Methods}

Incubation conditions. The incubation mixture, with a total volume of $200 \mu \mathrm{l}$, consisted of $50 \mathrm{mM}$ Tris-HCl buffer ( $\mathrm{pH} 7.4$ ), $5 \mathrm{mM} \mathrm{MgCl}_{2}, 0.2 \mathrm{mM}$ DTT, $0.2 \mathrm{mM}$ SAM and the liver S9 sample. In all of the experiments, 3-BTD was serially diluted to the required concentration and the final concentration of DMSO did not exceed $1 \%(\mathrm{v} / \mathrm{v})$ in the mixture. After pre-incubation at $37^{\circ} \mathrm{C}$ for $3 \mathrm{~min}$, the reaction was initiated by adding SAM and further incubated at $37^{\circ} \mathrm{C}$ in a shaking water bath. The reaction was terminated with the addition of ice-cold acetonitrile $(200 \mu \mathrm{l})$ containing $0.2 \%$ formic acid $(\mathrm{v} / \mathrm{v})$. The mixture was kept on ice until it was centrifuged at $20000 \times g$ for $10 \mathrm{~min}$ at $4{ }^{\circ} \mathrm{C}$. Aliquots of the supernatants were stored at $-20^{\circ} \mathrm{C}$ until analysis. Control incubations without S9 were carried out to ensure that the metabolite formation was S9-dependent. All incubations throughout the study were carried out in triplicate.

Chemical synthesis of 3-BTMD. $\mathrm{NaH}$ (60\% in oil, $0.16 \mathrm{~g}, 4.0$ $\mathrm{mmol})$ was slowly added to a solution of 3-BTD $(0.5 \mathrm{~g}, 1.6 \mathrm{mmol})$ in DMF $(15 \mathrm{ml})$ at $0{ }^{\circ} \mathrm{C}$ under dry argon and stirred for $0.5 \mathrm{~h}$. Methyl iodide $(0.26 \mathrm{ml}, 4.0 \mathrm{mmol})$ was added drop-wise to the reaction mixture at $0{ }^{\circ} \mathrm{C}$, maintaining the temperature at $0-5{ }^{\circ} \mathrm{C}$ for $4 \mathrm{~h}$. The reaction mixture was poured into water and ice (50 $\mathrm{ml}$ ) carefully and acidified using $2 \mathrm{~mol} \mathrm{l}^{-1}$ hydrochloric acid. The reaction mixture was extracted using ethyl acetate $(50 \mathrm{ml} \times$ $3)$. The combined organic layer was washed with water and brine, dried over anhydrous sodium sulfate, and concentrated via evaporation. The residue was purified using column chromatography with silica gel and $\mathrm{MeOH} / \mathrm{DCM}$ as the eluent to obtain 3-BTMD $(0.19 \mathrm{~g})$ in $38 \%$ yield as a yellow solid. Its structure was characterized using NMR techniques including ${ }^{1} \mathrm{H}-\mathrm{NMR},{ }^{13} \mathrm{C}-\mathrm{NMR}$, heteronuclear singular quantum correlation (HSQC) and heteronuclear multiple bond correlation (HMBC). The methylated product was stored at $-80{ }^{\circ} \mathrm{C}$ before dissolving in DMSO-d6 (Euriso-Top, Saint-Aubin, France) for NMR analysis. All experiments were carried out on a Bruker Avance-400 NMR spectrometer (Bruker, Switzerland). Chemical shifts were given on a $\delta$ scale and referenced against tetramethylsilane (TMS) at $0 \mathrm{ppm}$ for ${ }^{1} \mathrm{H}-\mathrm{NMR}(400 \mathrm{MHz})$ and ${ }^{13} \mathrm{C}-\mathrm{NMR}$ (150 MHz). ${ }^{1} \mathrm{H}$ NMR (400 MHz, DMSO) $\delta: 10.90$ (s, 1H), 9.14 (s, $1 \mathrm{H}), 8.16(\mathrm{~d}, J=8.0 \mathrm{~Hz}, 1 \mathrm{H}), 8.04(\mathrm{~d}, J=8.0 \mathrm{~Hz}, 1 \mathrm{H}), 7.67(\mathrm{~d}, J=$ $8.8 \mathrm{~Hz}, 1 \mathrm{H}), 7.56$ (t, $J=7.2 \mathrm{~Hz}, 1 \mathrm{H}), 7.45(\mathrm{t}, J=7.2 \mathrm{~Hz}, 1 \mathrm{H}), 7.00$ (d, $J=8.8 \mathrm{~Hz}, 1 \mathrm{H}), 3.90$ (s, 3H). ${ }^{13} \mathrm{C}$ NMR (100 MHz, DMSO) $\delta 160.80,159.97,156.45,152.48,148.66,143.40,136.16,134.47$, 126.99, 126.48, 125.51, 122.68, 122.60, 115.13, 115.08, 112.81, 61.23; HRMS (ESI) calcd for $\mathrm{C}_{17} \mathrm{H}_{10} \mathrm{NO}_{4} \mathrm{~S}^{-}:[\mathrm{M}-\mathrm{H}]^{-}: 324.0331$ found: 324.0335 .

Biosynthesis of metabolite and structural identification. The methylated metabolite of 3-BTD was biosynthesized using mixed liver S9 samples from the different species and purified for structure elucidation and quantitative analysis. In brief, 3BTD ( $1 \mathrm{mM})$ was incubated with mixed liver S9 $(0.5 \mathrm{mg}$ protein per ml), $50 \mathrm{mM}$ Tris-HCl (pH 7.4), $5 \mathrm{mM} \mathrm{MgCl}_{2}, 0.2 \mathrm{mM}$ DTT, and $0.2 \mathrm{mM}$ SAM to make $1 \mathrm{ml}$ of final incubation for $4 \mathrm{~h}$ at 37 ${ }^{\circ} \mathrm{C}$. This analytical-scale reaction was scaled up to a volume of $48 \mathrm{ml}$. The stock solution of 3-BTD $(100 \mathrm{mM})$ was prepared in DMSO. The concentration of the organic solvent in the final incubation was $1 \%$. The reaction was terminated with the addition of ice-cold methanol, and then the vessels were transferred to an ice bath and cooled for $20 \mathrm{~min}$. After the removal of the protein via centrifugation at $20000 \mathrm{~g}$ for $30 \mathrm{~min}$ at $4{ }^{\circ} \mathrm{C}$, the supernatants were collected and combined. The metabolite was separated from its untransformed substrate using preparative liquid chromatography performed on an Elite P2300 instrument with an Elite UV2300 detector and a Thermo C18 column $(250 \mathrm{~mm} \times 10 \mathrm{~mm}, 5 \mu \mathrm{m})$. The fraction of metabolite was collected and dried using a vacuum evaporator at $45{ }^{\circ} \mathrm{C}$. The methylated metabolite of 3-BTD was obtained as a powder and its ${ }^{1} \mathrm{H}-\mathrm{NMR}$ spectrum was obtained and compared to that of 3-BTMD. The NMR analysis was performed as mentioned above.

Kinetic analysis. The kinetic parameters of 3-BTD methylation in liver S9 from humans and the various animal species were determined under the incubation conditions in which the formation rates of 3-BTMD were in the linear range in relation to incubation time and protein concentration. Briefly, 3-BTD was incubated with the pooled HLS9 $(0.2 \mathrm{mg}$ protein per $\mathrm{ml})$, CyLS9 (0.2 mg protein per $\mathrm{ml})$, DLS9 $(0.2 \mathrm{mg}$ protein per $\mathrm{ml})$, MLS9 (0.2 $\mathrm{mg}$ protein per $\mathrm{ml})$, RLS9 (0.2 $\mathrm{mg}$ protein per $\mathrm{ml})$, PLS9 (0.2 $\mathrm{mg}$ protein per $\mathrm{ml})$, GpLS9 ( $0.2 \mathrm{mg}$ protein per $\mathrm{ml}$ ) and RaLS9 (0.2 $\mathrm{mg}$ protein per $\mathrm{ml}$ ) for $5 \mathrm{~min}$, separately. The enzymatic kinetic parameters were estimated via nonlinear regression using GraphPad Prism software, version 6.0. The apparent $K_{\mathrm{m}}$ and $V_{\max }$ values were calculated from the nonlinear regression analysis of the experimental data according to the 
Michaelis-Menten equation, and the results were graphically represented using Eadie-Hofstee plots, where $K_{\mathrm{m}}$ is the substrate concentration corresponding to the $1 / 2 V_{\max }$.

For the Michaelis-Menten kinetic model:

$$
v=\frac{V_{\max } \times[\mathrm{S}]}{K_{\mathrm{m}}+[\mathrm{S}]}
$$

where $v$ is the rate of reaction, $[\mathrm{S}]$ is the substrate concentration, $V_{\text {max }}$ is the maximum velocity estimate, and $K_{\mathrm{m}}$ is the apparent affinity constant.

The intrinsic clearances $\left(\mathrm{Cl}_{\text {int }}\right) \quad\left(V_{\max } / K_{\mathrm{m}}\right)$ were also calculated.

Chemical inhibition assays. Chemical inhibition assays of 3BTD methylation in liver S9 from humans and the animal species were conducted using a selective COMT inhibitor (tolcapone). Tolcapone (0-1000 nM) was pre-incubated with the reaction mixtures containing $50 \mathrm{mM}$ Tris- $\mathrm{HCl}$ buffer ( $\mathrm{pH} 7.4$ ), $5 \mathrm{mM} \mathrm{MgCl}_{2}, 0.2 \mathrm{mM}$ DTT, $0.2 \mathrm{mM}$ SAM, 3-BTD $(1 \mu \mathrm{M})$ and the liver S9 $\left(0.2 \mathrm{mg} \mathrm{ml}^{-1}\right)$ at $37^{\circ} \mathrm{C}$ for $10 \mathrm{~min}$, separately. Then the reaction was started with the addition of SAM. Moreover, the inhibitory effects of tolcapone (0-1000 nM) towards the 3-BTD methylation in liver S9 of humans and the animal species were investigated. The $\mathrm{IC}_{50}$ values were also determined.

Fluorescence detection and LC-ESI-MS analysis. All fluorescence-based assays for 3-BTMD were performed on a Synergy H1 Hybrid Multi-Mode Microplate Reader (BioTek) with fluorescence emission at $510 \mathrm{~nm}$ and fluorescence absorbance at $390 \mathrm{~nm}$ (gain = 100).

The UFLC system was equipped with a CBM-20A Communications Bus Module, an SIL-20ACHT autosampler, two LC20AD pumps, a DGU-20A3 vacuum degasser, a CTO-20AC column oven and an SPD-M 20A diode array detector (Kyoto, Japan). A Shim-pack XR-ODS (750 mm $\times 2.0 \mathrm{~mm}, 2.2 \mathrm{~mm}$, Shimadzu, Kyoto, Japan) analytical column with an ODS guard column $(5 \mathrm{~mm} \times 2.0 \mathrm{~mm}, 2.2 \mu \mathrm{m}$, Shimadzu) was used to separate 3-BTD and its metabolite. The mobile phase consisted of $\mathrm{CH}_{3} \mathrm{CN}(\mathrm{A})$ and water containing $0.2 \%(\mathrm{v} / \mathrm{v})$ formic acid (B), with the following gradient profile: $0-10 \mathrm{~min}, 90-10 \% \mathrm{~B} ; 10-$ $13 \mathrm{~min}, 5 \% \mathrm{~B}$; $13-16 \mathrm{~min}$, balanced to $90 \% \mathrm{~B}$. The flow rate was $0.4 \mathrm{ml} \mathrm{min}{ }^{-1}$ and the column temperature was kept at $40{ }^{\circ} \mathrm{C} .3-$ BTD and its metabolite 3-BTMD were detected at $390 \mathrm{~nm}$.

A Shimadzu LC-MS-2010EV (Kyoto, Japan) instrument with an ESI interface was used for the identification of 3-BTD and its metabolite. Mass detection was performed in both the positiveion mode $\left(\mathrm{ESI}^{+}\right)$and negative ion mode $\left(\mathrm{ESI}^{-}\right)$from $\mathrm{m} / \mathrm{z} 100$ to 800. The detector voltage was set at $+1.75 \mathrm{kV}$ and $-1.55 \mathrm{kV}$ for positive and negative ion detection, respectively. The curved desolvation line temperature (CDL) and the block heater temperature were both set at $250{ }^{\circ} \mathrm{C}$. Other MS detection conditions were as follows: interface voltage, $4 \mathrm{kV}$; CDL voltage, $40 \mathrm{~V}$; nebulizing gas $\left(\mathrm{N}_{2}\right)$ flow, $1.51 \mathrm{~min}^{-1}$; drying gas $\left(\mathrm{N}_{2}\right)$ pressure, 0.06 $\mathrm{MPa}$. Data processing was performed using LCMS Solution version 3.41 software (Kyoto, Japan).

\section{Conflicts of interest}

There are no conflicts to declare.

\section{Acknowledgements}

This work was supported by NSF of China (81603187 \& 31600641).

\section{References}

1 J. Axelrod and R. Tomchick, J. Biol. Chem., 1958, 233(3), 702705.

2 H. C. Guldberg and C. A. Marsden, Pharmacol. Rev., 1975, 27(2), 135-206.

3 T. Ellingson, S. Duddempudi, B. D. Greenberg, D. Hooper and G. Eisenhofer, J. Chromatogr. B: Biomed. Sci. Appl., 1999, 729(1-2), 347-353.

4 E. M. Tunbridge, P. J. Harrison and D. R. Weinberger, Biol. Psychiatry, 2006, 60(2), 141-151.

5 C. B. Meloto, S. K. Segall, S. Smith, M. Parisien, S. A. Shabalina, C. M. Rizzatti-Barbosa, J. Gauthier, D. Tsao, M. Convertino, M. H. Piltonen, G. D. Slade, R. B. Fillingim, J. D. Greenspan, R. Ohrbach, C. Knott, W. Maixner, D. Zaykin, N. V. Dokholyan, I. Reenilä, P. T. Männistö and L. Diatchenko, Pain, 2015, 156(10), 2072-2083.

6 R. M. Bilder, J. Volavka, H. M. Lachman and A. A. Grace, Neuropsychopharmacology, 2004, 29(11), 1943-1961.

7 E. L. Cavalieri and E. G. Rogan, J. Steroid Biochem. Mol. Biol., 2011, 125(3-5), 169-180.

8 B. T. Zhu, E. L. Ezell and J. G. Liehr, J. Biol. Chem., 1994, 269(1), 292-299.

9 D. Muguet, E. Broussolle and G. Chazot, Biomed. Pharmacother., 1995, 49(4), 197-209.

10 R. D. Strous, N. Bark, S. S. Parsia, J. Volavka and H. M. Lachman, Psychiatry Res., 1997, 69(2-3), 71-77.

11 A. Vercammen, C. S. Weickert, A. J. Skilleter, R. Lenroot, P. R. Schofield and T. W. Weickert, Transl. Psychiatry, 2014, 4, e356.

12 P. Wang, Y. L. Xia, L. W. Zou, X. K. Qian, T. Y. Dou, Q. Jin, S. Y. Li, Y. Yu, D. D. Wang, Q. Luo, G. B. Ge and L. Yang, Chemistry, 2017, 23(45), 10800-10807.

13 C. Marin and J. A. Obeso, Int. Rev. Neurobiol., 2010, 95, 191205.

14 O. Kambur, R. Talka, O. B. Ansah, V. K. Kontinen, A. Pertovaara, E. Kalso and P. T. Männistö, Br. J. Pharmacol., 2010, 161(7), 1553-1565.

15 A. Tomlinson, B. Grayson, S. Marsh, A. Hayward, K. M. Marshall and J. C. Neill, Eur. Neuropsychopharmacol., 2015, 25(4), 454-467.

16 M. F. Egan, T. E. Goldberg, B. S. Kolachana, J. H. Callicott, C. M. Mazzanti, R. E. Straub, D. Goldman and D. R. Weinberger, Proc. Natl. Acad. Sci. U. S. A., 2001, 98(12), 6917-6922.

17 M. A. Vieira-Coelho, P. Gomes, M. P. Serrão and P. Soares-daSilva, Kidney Int., 2001, 59(5), 1683-1694.

18 A. A. Sharif, Mov. Disord., 2002, $17(2), 421$.

19 M. Berk, S. Dodd, M. Kauer-Sant'Anna, G. S. Malhi, M. Bourin, F. Kapczinski and T. Norman, Acta Psychiatr. Scand., Suppl., 2007, 434, 41-49. 
20 N. Jatana, N. Apoorva, S. Malik, A. Sharma and N. Latha, Cent. Nerv. Syst. Agents Med. Chem., 2013, 13(3), 166-194.

21 L. E. Kiss and P. Soares-da-Silva, J. Med. Chem., 2014, 57(21), 8692-8717.

22 O. Pelkonen, M. Turpeinen, J. Uusitalo, A. Rautio and H. Raunio, Basic Clin. Pharmacol. Toxicol., 2005, 96(3), 167175.

23 X. C. Ma, J. Ning, G. B. Ge, S. C. Liang, X. L. Wang, B. J. Zhang, S. S. Huang, J. K. Li and L. Yang, Drug Metab. Dispos., 2011, 39(4), 675-682.

24 G. B. Ge, C. Z. Ai, W. B. Hu, J. Hou, L. L. Zhu, G. Y. He, Z. Z. Fang, S. C. Liang, F. Y. Wang and L. Yang, Eur. J. Pharm. Sci., 2013, 48(1-2), 360-369.

25 J. Ning, J. Hou, P. Wang, J. J. Wu, Z. R. Dai, L. W. Zou, W. Li, G. B. Ge, X. C. Ma and L. Yang, Xenobiotica, 2015, 45(11), 954-960.

26 H. Ong and N. Yamaguchi, Can. J. Physiol. Pharmacol., 1982, 60(5), 735-737.
27 K. Haasio, A. Koponen, K. E. Penttila and E. Nissinen, Eur. J. Pharmacol., 2002, 453(1), 21-26.

28 M. A. Vieira-Coelho and P. Soares-da-Silva, Br. J. Pharmacol., 1996, $117(3)$, 516-520.

29 C. De Santi, P. C. Giulianotti, A. Pietrabissa, F. Mosca and G. M. Pacifici, Eur. J. Clin. Pharmacol., 1998, 54(3), 215-219.

30 H. W. Bai, J. Y. Shim, J. Yu and B. T. Zhu, Chem. Res. Toxicol., 2007, 20(10), 1409-1425.

31 Z. Ma, H. Liu and B. Wu, Br. J. Clin. Pharmacol., 2014, 77(3), 410-420.

32 J. Vidgren, L. A. Svensson and A. Liljas, Nature, 1994, 368(6469), 354-358.

33 T. Lotta, J. Vidgren, C. Tilgmann, I. Ulmanen, K. Melén, I. Julkunen and J. Taskinen, Biochemistry, 1995, 34(13), 4202-4210.

34 A. J. Shield, B. A. Thomae, B. W. Eckloff, E. D. Wieben and R. M. Weinshilboum, Mol. Psychiatry, 2004, 9(2), 151-160. 